\title{
MRI Reporter Genes
}

\author{
Assaf A. Gilad ${ }^{1}$, Keren Ziv², Michael T. McMahon ${ }^{1,3}$, Peter C.M. van Zij1 ${ }^{1,3}$, Michal Neeman², and Jeff W.M. Bulte ${ }^{1}$ \\ ${ }^{1}$ Johns Hopkins University School of Medicine, Baltimore, Maryland; ${ }^{2}$ The Weizmann Institute of Science, Rehovot, Israel; and ${ }^{3}$ Hugo \\ Moser Kennedy Krieger Research Institute, Baltimore, Maryland
}

\begin{abstract}
Noninvasive molecular imaging of dynamic processes has benefited tremendously from the use of reporter genes. These genes encode for proteins that emit light, bind radiolabeled probes, or, as covered in this review, modulate MRI contrast. Reporter genes play a pivotal role in monitoring cell trafficking, gene replacement therapy, protein-protein interactions, neuronal plasticity, and embryonic development. Several strategies exist for generating MRI contrast: using enzyme-catalyzed chemical modification of metal-based contrast agents or (phosphorus) metabolites, iron-binding and iron-storage proteins to accumulate iron as a contrast agent, and artificial proteins for imaging based on chemical exchange saturation transfer. MRI reporter genes have the advantage that the specific signal can be coregistered with soft-tissue anatomy and functional tissue information and have, therefore, become an active and growing area of scientific interest.
\end{abstract}

Key Words: molecular imaging; MR imaging; MRI spectroscopy; reporter gene; ferritin

J Nucl Med 2008; 49:1905-1908

DOI: 10.2967/jnumed.108.053520

With more than 20,000 genes in the human genome now identified and a similar number of genes in the rat and mouse genome known (1), explanation of the function of genes has become the major challenge. Clarification of the function of genes has been facilitated by the development of reporter genes, which are revolutionizing the way many current biomedical studies are designed and performed. A reporter gene is a gene whose product can be readily detected and either be fused to the gene of interest or can replace it. The main applications for these reporters include monitoring gene expression levels, investigating dynamic molecular interactions between proteins, studying cellular interactions, tracking cells in normal and abnormal development or in cell transplantation therapy, and monitoring gene replacement therapy.

Optical reporter genes are probably the most commonly used for imaging and widely developed. Throughout the years, multiple genes were cloned from a variety of organisms that emit light via bioluminescence or fluorescence in multiple distinguishable wave-

Received Jun. 20, 2008; revision accepted Sep. 15, 2008.

For correspondence or reprints contact: Assaf A. Gilad, Department of Radiology, Johns Hopkins University School of Medicine, 1550 Orleans St., Cancer Research Building II, Room 4M63, Baltimore, MD 21231.

E-mail: assaf@mri.jhu.edu

COPYRIGHT ๑ 2008 by the Society of Nuclear Medicine, Inc. lengths. Probably the most recent and exciting implementation of optical reporter genes was the development of the transgenic "brainbow" mouse, in which combinatorial expression of fluorescent proteins in the mouse brain resulted in the production of more than 89 distinguishable colors (2). An emerging new class of reporter genes encodes for proteins with affinity for radioisotopes or positron emitter probes. These receptors, transporters, or enzymes can provide quantitative images on administration of suitable radiolabeled probes (3). MRI reporter genes are unique among all reporter genes used with the various imaging modalities because they can provide information on gene expression that can be coregistered with anatomic and functional information (4). MRI reporters enable serial temporal imaging within the same subject. This is particularly useful for studying dynamic processes, for example, migration of stem cells and progenitors (5), neuronal plasticity, mechanisms of development and adaptation, disease progression and response to trauma or illness, and processes of memory and learning.

The purpose of this review was to give a basic, brief overview of the recent developments in the field of MRI reporter genes and to evaluate and compare the currently existing genes (Table 1) while suggesting future directions. A more detailed review can be found in Gilad et al. (4) and Modo et al. (6). For further reading, the context of MRI reporter genes is closely linked to certain MRI contrast agents that can act as sensors for changes in biologic or physiologic conditions (7).

\section{BASIC PRINCIPLES OF MRI CONTRAST}

MRI relies on measuring magnetization of magnetic nuclei subjected to radiofrequency irradiation inside a magnetic field. A variety of nuclei can be studied, with the most common one being the hydrogen nucleus (proton, ${ }^{1} \mathrm{H}$ ). For example, MRI detects properties of the water signal, mostly in terms of amount of water (so-called proton or spin density), its relaxation back to equilibrium after excitation (time constant, T1), and its signal broadening (relaxation times, T2 for natural signal width, and $\mathrm{T} 2 *$, which includes T2 effects plus magnetic field inhomogeneity contributions). Any tissue difference in terms of these properties provides contrast. Also, besides water, MR spectroscopy (MRS) can detect individual proton signals in metabolites and nuclei different from protons, such as ${ }^{31} \mathrm{P},{ }^{13} \mathrm{C},{ }^{23} \mathrm{Na},{ }^{19} \mathrm{~F}$, and many more, all of which have specific frequencies. The signal acquired from different nuclei depends on their abundance in the tissue and their chemical interaction with other molecules, allowing multiple manipulations with various MRI acquisition schemes to create different contrasts. When certain metals or organic compounds are present, endogenous contrast mechanisms may be affected. T1 relaxation can be enhanced using compounds containing paramagnetic lanthanides, 
TABLE 1

Comparison of Existing MR Reporter Genes

\begin{tabular}{|c|c|c|c|c|}
\hline Gene & Contrast & Substrate & Advantages & Disadvantages \\
\hline CK & ${ }^{31} \mathrm{P}-\mathrm{MRS}$ & $\begin{array}{l}\text { Endogenous adenosine } \\
\text { triphosphate }\end{array}$ & $\begin{array}{l}\text { No background signal } \\
\text { in most tissues }\end{array}$ & Spectroscopic image (low resolution) \\
\hline Tyrosinase & $\mathrm{T} 2 / \mathrm{T}^{*}$ & $\begin{array}{l}\text { Endogenous or exogenous } \\
\text { iron }\end{array}$ & $\begin{array}{l}\text { Rapid straightforward } \\
\text { gene product }\end{array}$ & Signal dependent on availability of iron \\
\hline$\beta$-gal & T1 & Exogenous EgadMe & Positive contrast & $\begin{array}{l}\text { Exogenous agent also gives partial } \\
\text { signal without reporter gene }\end{array}$ \\
\hline Transferrin receptor & $\mathrm{T} 2 / \mathrm{T}^{*}$ & Exogenous transferrin-MION & High sensitivity & $\begin{array}{l}\text { Accessibility and nonspecific uptake of } \\
\text { substrate nanoparticles }\end{array}$ \\
\hline Ferritin & $\mathrm{T} 2 / \mathrm{T} 2 *$ & $\begin{array}{l}\text { Endogenous or exogenous } \\
\text { iron }\end{array}$ & High sensitivity & $\begin{array}{l}\text { Delay of change in signal that is } \\
\text { dependent on iron availability } \\
\text { and ferritin loading factor }\end{array}$ \\
\hline Mag A & T2/T2* & $\begin{array}{l}\text { Endogenous or exogenous } \\
\text { iron }\end{array}$ & High sensitivity & $\begin{array}{l}\text { Delay of change in signal that is } \\
\text { dependent on iron availability }\end{array}$ \\
\hline LRP & CEST & None & $\begin{array}{c}\text { Multicolor detection, } \\
\text { on-off switchable }\end{array}$ & Current low sensitivity \\
\hline
\end{tabular}

with gadolinium being widely used in the clinic. Iron oxide particles, on the other hand, can drastically shorten T2 and T2*, which is manifested by darkening of the MR images. As a consequence, many iron-binding proteins are potential candidates for MRI reporter genes.

Chemical exchange saturation transfer (CEST) is a new type of MRI contrast that has been developed; it relies on the chemical exchange of protons of solutes such as contrast agents with bulk water (8). A variety of organic and organometallic compounds have a sufficient number of protons with suitable chemical exchange rates and specific MRI frequencies to be excited selectively and detected sensitively. A radiofrequency pulse, called a saturation pulse, is applied at the exchangeable proton resonance frequency. This saturation, or signal loss, is then transferred via exchange to bulk water, producing a fractional reduction in the water signal (Figs. 1A-1D).

\section{MRS: ENZYME-BASED REPORTER GENES}

Historically, the first MRI reporter gene was creatine kinase (CK), an enzyme that catalyzes adenosine triphosphate conversion to adenosine diphosphate-producing phosphocreatine $(\mathrm{PCr})$ and can be detected by ${ }^{31} \mathrm{P}$ MRS. The first demonstration of CK was in its transgenic overexpression in the liver (9), in which high levels of $\mathrm{PCr}$ were observed; $\mathrm{PCr}$, however, is absent in the livers of control mice. CK was subsequently used as a marker of low-density lipoprotein receptor (LDLr) expression in LDLr-deficient mice after treatment with an adenovirus engineered to coexpress recombinant human LDLr and the CK-B reporter gene (10). In a recent study, MRS was used to examine different promoters in yeast cells. Genes that catalyze the formation of polyphosphate were cloned and placed after different promoters, after which polyphosphate levels could be monitored with ${ }^{31} \mathrm{P}$ MRI (11).
FIGURE 1. Principles of CEST contrast mechanism. Frequency-selective saturation pulse is applied to label amide protons ( $A$, green) or guanidyl protons $(C, r e d)$ of polypeptide contrast agent. Labeled protons exchange with water protons, which leads to reduction in MRI signal intensity $(\Delta \mathrm{SI})$ in frequency-selective manner (B and D). CEST maps of MRI phantoms were acquired with frequency-selective saturation pulses (E) at $\pm 3.7 \mathrm{ppm}$, which enhances PLL $(F)$; at $\pm 1.8 \mathrm{ppm}$, which enhances PLA (G); and at $\pm 0.8 \mathrm{ppm}$, which enhances PLT $(\mathrm{H})$. Merged composition image of maps $(\mathrm{F}-\mathrm{H})$ is shown in I. PBS = phosphate-buffered saline. (Modified from McMahon et al. (23) and Gilad et al. (24)).
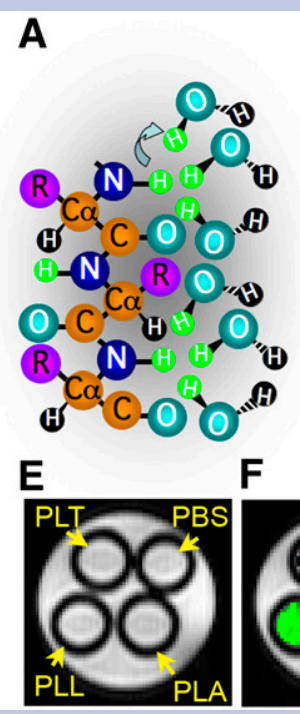

B

C

Excitation

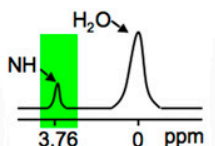

Detection

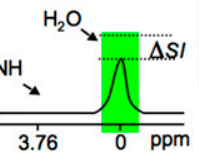

G

$F$

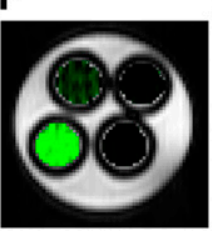

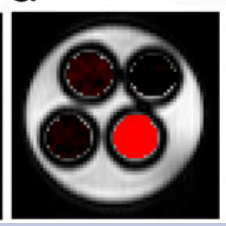

D

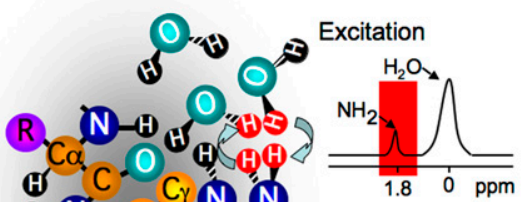

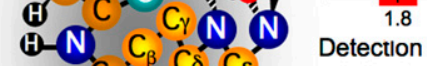

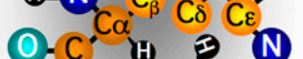

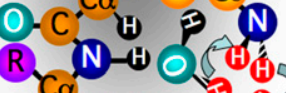
${ }^{\mathrm{Ca}} \mathrm{C}-\mathrm{O}$

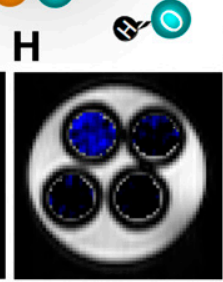

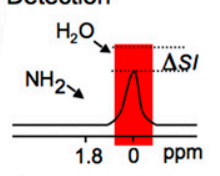

I

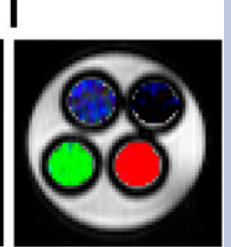




\section{MRI: ENZYME-BASED REPORTER GENES}

The overexpression of human tyrosinase initiates a chain of reactions including melanin production, which is followed by higher metal binding that may result in enhanced MRI signal intensity. A tetracycline-inducible system of tyrosinase has been developed in MCF-7 breast cancer cells (12) using this concept.

Another enzyme that was exploited as an MRI reporter gene is $\beta$-galactosidase ( $\beta$-gal). $\beta$-gal is an enzyme encoded by the LacZ gene of Escherichia coli; it catalyzes hydrolysis of $\beta$-D-galactosides and is widely used in microscopy because of its large variety of substrates. The use of $\beta$-gal in MRI was demonstrated by its effect on (1-(2-( $\beta$-galactopyranosyloxy)propyl)-4,7,10-tris(carboxymethyl)1,4,7,10-tetraazacyclododecane)gadolinium(III) (EgadMe) (13). Once EgadMe was cleaved by $\beta$-gal and its free coordination site of gadolinium was exposed, it altered the relaxation time of water and created contrast. $\beta$-gal was also used in spectroscopic imaging (14).

\section{MRI: IRON-BASED REPORTER GENES}

Another possibility for creating MRI reporter genes is the expression of iron-related proteins. Iron, depending on its oxidation state and its structure, can act as a paramagnetic reactive metal, which is essential for body homeostasis. The manipulation of iron concentration is expected to yield a detectable change in MRI contrast, which will be able to produce a real-time report on cellular events. Therefore, several iron-related proteins were tested as possible MRI reporters.

In the bloodstream, iron is bound to the plasma protein transferrin and enters cells via interaction of the iron-transferrin complex with the transferrin receptor. Administration of transferrin linked to monocrystalline iron-oxide nanocompounds to nude mice that had hTfR-transfected 9-L gliosarcoma tumors led to a significant difference in MRI signal $24 \mathrm{~h}$ after administration (15). The transferrin receptor has also been used for monitoring therapeutic gene expression when expressed as part of a vector that carried several genes, among them a prodrug therapy gene. All 3 transgenes were expressed in the same cell simultaneously, and MRI demonstrated the ability to use transferrin receptor as a reporter for gene therapy (16).

Although iron has a crucial role in body homeostasis, excess of iron can cause damage to cells by catalyzing the Fenton reaction, which generates reactive oxygen species. Therefore, excess intra- cellular iron is stored in the ferrihydrite core of the ferritin protein. The first use of ferritin in the design of C6 glioma MRI reporter cells was done with a construct that carried enhanced green fluorescent protein and the heavy chain of ferritin, which exhibits ferroxidase activity, under tetracycline control. Inoculation of these tumor cells in nude mice revealed a significant decrease in $\mathrm{T} 2$ on tetracycline withdrawal (17). Transgenic mice that expressed the heavy chain of ferritin in a tissue-specific and tetracycline-dependent manner revealed a significant change in $\mathrm{T} 2$ values that was tissuedependent: decreased $\mathrm{T} 2$ when expressed exclusively by endothelial cells as expected (Fig. 2) and surprisingly increased T2 when expressed by liver hepatocytes. The reason for the opposite direction of $\mathrm{T} 2$ changes in the liver was found to be related to the overexpression of H-ferritin in the hepatocytes, which induced mild toxicity and resulted in water vacuole formation. These water vacuoles contributed to the elevation in $\mathrm{T} 2$ values due to the long T2 of free water (18).

Genove et al. demonstrated the use of ferritin as an MRI reporter gene by injecting an adenovirus that encoded for human ferritin into the brain parenchyma of mice, resulting in significant loss of signal at the site of inoculation (19). Coexpression of ferritin and the transferrin receptor showed signal loss on T2- and T2*-weighted MR images of neuronal stem cells in an iron-enriched environment (20). Further improvements in ferritin MRI reporter gene sensitivity may be possible. For instance, Bennett et al. proposed that aggregation of ferritin could augment its efficacy. Chemical crosslinking of ferritin to actin in vitro led to polymerization, which resulted in a $20 \%$ decrease in T2 (21).

Recently, Mag A, a bacterial iron transporter in Aquaspirillum magnetotacticum from the magnetotactic bacteria family, was expressed in 293FT cells under doxycycline regulation. Administration of doxycycline and iron resulted in significantly shorter $\mathrm{T} 2$, and inoculation of these cells to a mouse brain induced significant signal loss on $\mathrm{T} 2 *$-weighted images (22).

\section{MRI: CEST-BASED REPORTER GENES}

CEST contrast agents have 2 major advantages. First, they are switchable; that is, the contrast is detectable only when a saturation pulse is applied at the specific frequency characteristic of the exchangeable protons of an agent. Otherwise, the contrast agent is MRI-invisible. The second advantage is that different contrast agents with different excitation frequencies can be used for imaging simultaneously more than one target. This property might be

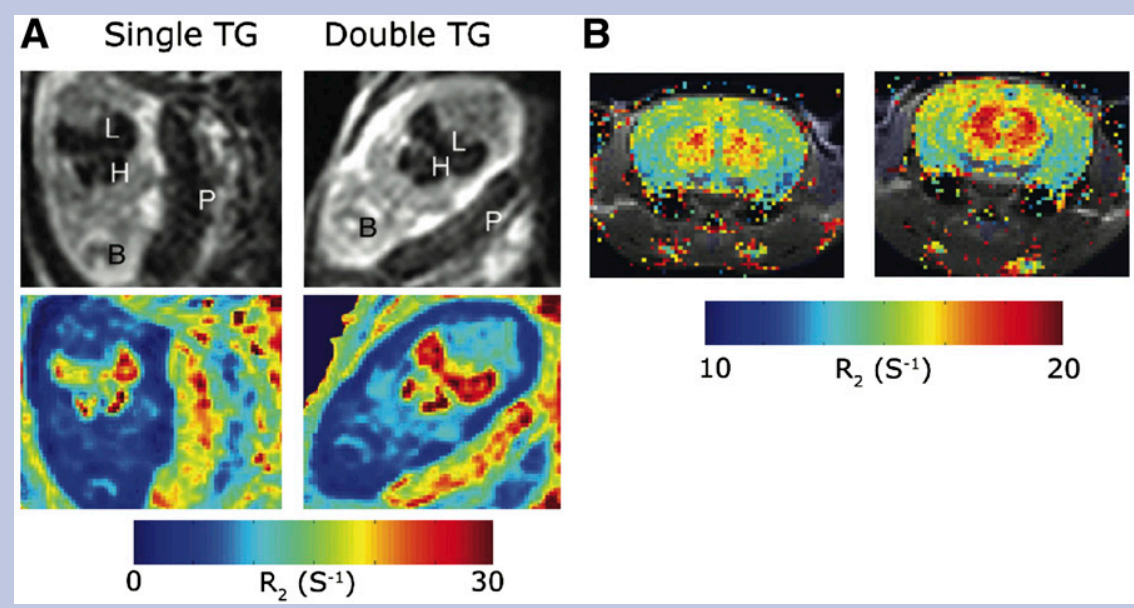

FIGURE 2. MRI detection of endothelial overexpression of $\mathrm{H}$-ferritin in transgenic mice. Expression of ferritin was regulated by vascular endothelial cadherin promoter in double transgenic mice but not in single transgenic siblings. Elevated $R^{2}$ was observed in liver and heart of E13.5 embryos studied in utero (A) and in brain of adult mice (B). TG = transgenic. (Adapted from Cohen et al. (18)). 
exploited for imaging multiple cells (e.g., for studying neuronal circuits) or the expression of multiple genes (such as in genetic circuits). This so-called multicolor CEST MRI concept is shown in Figures $1 \mathrm{E}-1 \mathrm{H}$ for the polypeptides poly-L-lysine (PLL), poly-Larginine (PLA), and poly-L-threonine (PLT) (23). Although PLL is detectable through its exchangeable amide protons, PLA provides strong CEST contrast through the exchangeable guanidyl protons and PLT has exchangeable hydroxyl protons, with a unique frequency. Although each of the polypeptides provides some CEST contrast in the frequency range of other polypeptides, it is relatively easy to separate the signal derived from each polypeptide with proper image processing. Therefore, the 3 different polypeptides are clearly distinguishable with MRI, making them suitable candidates for labeling different targets.

As a first demonstration, a lysine-rich protein (LRP), containing a high density of amide protons, was cloned and expressed in 9-L rat glioma cells. The LRP amino acid sequence is similar to PLL and provides MRI contrast at the amide proton frequency. LRP overexpression was detectable in vitro in cell extracts and in vivo in xenografts of LRP-expressing 9-L cells in the mouse brain (24).

\section{FUTURE PROSPECTS}

MRI reporter genes are still in their infancy and need to be further improved to facilitate wider use. A major improvement in sensitivity for both ferritin and CEST reporter genes is likely to occur with MRI scanners moving toward ever higher fields (up to $9.4 \mathrm{~T}$ for humans and as high as $18.8 \mathrm{~T}$ for scanners suitable for animal research). In addition, the acquisition of more specific reporter-induced MRI contrast may be achieved using optimized pulse sequences and improved ways of image processing (analogous to those used for detecting small changes in blood oxygen level-dependent functional MRI). From the molecular biology side, reporter genes may be improved through directed evolution in a fashion similar to that accomplished for the fluorescent reporter genes (25). A key challenge would be to resolve multiple targets while performing simultaneous imaging. This could include different reporters driven by different promoters or developing sophisticated data-processing algorithms to distinguish gene expression temporally as well as spatially.

From the clinical point of view, a few significant hurdles still remain before reporter genes can be introduced into the human body. Once this has been achieved in a safe manner, we can foresee 2 major clinical applications. The first one will be tracking genetically labeled immunotherapeutic or regenerative stem cells for monitoring cell survival, migration or homing, and differentiation. The second application will be monitoring gene replacement therapy; here, the MRI reporter genes might become the method of choice to monitor successfully induced therapeutic gene expression long before a phenotypic readout becomes available.

\section{ACKNOWLEDGMENTS}

Drs. Gilad and Ziv contributed equally to this work. The studies described here were supported, in part, by grant KO1 EB006394 and grants from the Israel Science Foundation, the Minerva Foundation, and NIH Roadmap (R21 EB005252).

\section{REFERENCES}

1. Gibbs RA, Weinstock GM, Metzker ML, et al. Genome sequence of the Brown Norway rat yields insights into mammalian evolution. Nature. 2004;428:493-521.

2. Livet J, Weissman TA, Kang H, et al. Transgenic strategies for combinatorial expression of fluorescent proteins in the nervous system. Nature. 2007;450:56-62.

3. Serganova I, Ponomarev V, Blasberg R. Human reporter genes: potential use in clinical studies. Nucl Med Biol. 2007;34:791-807.

4. Gilad AA, Winnard PT Jr, van Zijl PC, Bulte JW. Developing MR reporter genes: promises and pitfalls. NMR Biomed. 2007;20:275-290.

5. Ben-Hur T, van Heeswijk RB, Einstein O, et al. Serial in vivo MR tracking of magnetically labeled neural spheres transplanted in chronic EAE mice. Magn Reson Med. 2007;57:164-171.

6. Modo MMJJ, Bulte JWM, eds. Molecular and Cellular MR Imaging. Boca Raton, FL: CRC Press; 2007.

7. Jasanoff A. MRI contrast agents for functional molecular imaging of brain activity. Curr Opin Neurobiol. 2007;17:593-600.

8. Sherry AD, Woods M. Chemical exchange saturation transfer contrast agents for magnetic resonance imaging. Annu Rev Biomed Eng. 2008;10:391-411.

9. Koretsky AP, Brosnan MJ, Chen LH, Chen JD, Van Dyke T. NMR detection of creatine kinase expressed in liver of transgenic mice: determination of free ADP levels. Proc Natl Acad Sci USA. 1990;87:3112-3116.

10. Li Z, Qiao H, Lebherz C, et al. Creatine kinase, a magnetic resonance-detectable marker gene for quantification of liver-directed gene transfer. Hum Gene Ther. 2005; 16:1429-1438.

11. Ki S, Sugihara F, Kasahara K, et al. A novel magnetic resonance-based method to measure gene expression in living cells. Nucleic Acids Res. 2006;34:e51.

12. Alfke H, Stoppler H, Nocken F, et al. In vitro MR imaging of regulated gene expression. Radiology. 2003;228:488-492.

13. Louie AY, Huber MM, Ahrens ET, et al. In vivo visualization of gene expression using magnetic resonance imaging. Nat Biotechnol. 2000;18:321-325.

14. Kodibagkar VD, Yu J, Liu L, Hetherington HP, Mason RP. Imaging betagalactosidase activity using ${ }^{19} \mathrm{~F}$ chemical shift imaging of LacZ gene-reporter molecule 2-fluoro-4-nitrophenol-beta-D-galactopyranoside. Magn Reson Imaging. 2006;24:959-962.

15. Weissleder R, Moore A, Mahmood U, et al. In vivo magnetic resonance imaging of transgene expression. Nat Med. 2000;6:351-355.

16. Ichikawa T, Hogemann D, Saeki Y, et al. MRI of transgene expression: correlation to therapeutic gene expression. Neoplasia. 2002;4:523-530.

17. Cohen B, Dafni H, Meir G, Harmelin A, Neeman M. Ferritin as an endogenous MRI reporter for noninvasive imaging of gene expression in C6 glioma tumors. Neoplasia. 2005;7:109-117.

18. Cohen B, Ziv K, Plaks V, et al. MRI detection of transcriptional regulation of gene expression in transgenic mice. Nat Med. 2007;13:498-503.

19. Genove G, DeMarco U, Xu H, Goins WF, Ahrens ET. A new transgene reporter for in vivo magnetic resonance imaging. Nat Med. 2005;11:450-454.

20. Deans AE, Wadghiri YZ, Bernas LM, Yu X, Rutt BK, Turnbull DH. Cellular MRI contrast via coexpression of transferrin receptor and ferritin. Magn Reson Med. 2006;56:51-59.

21. Bennett KM, Shapiro EM, Sotak CH, Koretsky AP. Controlled aggregation of ferritin to modulate MRI relaxivity. Biophys J. 2008;95:342-351.

22. Zurkiya O, Chan AW, Hu X. MagA is sufficient for producing magnetic nanoparticles in mammalian cells, making it an MRI reporter. Magn Reson Med. 2008;59:1225-1231.

23. McMahon MT, Gilad AA, DeLiso MA, Cromer Berman SM, Bulte JWM, van Zijl PCM. New "multicolor" polypeptide diamagnetic chemical exchange saturation transfer (DIACEST) contrast agents for MRI. Magn Reson Med. 2008;60:803-812.

24. Gilad AA, McMahon MT, Walczak P, et al. Artificial reporter gene providing MRI contrast based on proton exchange. Nat Biotechnol. 2007;25:217-219.

25. Shaner NC, Campbell RE, Steinbach PA, Giepmans BN, Palmer AE, Tsien RY. Improved monomeric red, orange and yellow fluorescent proteins derived from Discosoma sp. red fluorescent protein. Nat Biotechnol. 2004;22:1567-1572. 